\title{
Efficient Visible Photoluminescence from Carbon Nanotubes in Zeolite Templates
}

\author{
Jiandong Guo, ${ }^{1}$ Chunlei Yang, ${ }^{1}$ Z. M. Li,${ }^{1}$ Ming Bai, ${ }^{1}$ H. J. Liu,,${ }^{1}$ G. D. Li,${ }^{1}$ E. G. Wang, ${ }^{2}$ C. T. Chan, ${ }^{1}$ Z. K. Tang, ${ }^{1}$ \\ W. K. Ge, ${ }^{1}$ and Xudong Xiao,* \\ ${ }^{1}$ Department of Physics and Institute of Nano Science and Technology, Hong Kong University of Science and Technology, \\ Hong Kong, China \\ ${ }^{2}$ Institute of Physics, Chinese Academy of Science, Beijing, China
}

(Received 8 April 2003; published 30 June 2004)

\begin{abstract}
We have found strong visible photoluminescence (PL) from $0.4 \mathrm{~nm}$ carbon nanotubes embedded in the ordered channels of a zeolite crystal matrix. The PL exhibits a broad emission band with an efficiency of $\sim 1 \%-5 \%$. Strong polarization dependence is observed both on the excitation and emission, indicating a preferential optical dipole along the axis of carbon nanotubes. By correlating with the absorption and Raman spectra, we identify $(3,3)$ and $(4,2)$ tubes to be responsible for the PL peaks at $\sim 2.6$ and $\sim 1.7 \mathrm{eV}$, respectively.
\end{abstract}

DOI: 10.1103/PhysRevLett.93.017402

PACS numbers: 78.67.Ch, 61.46.+w, 78.55.Hx

Single-walled carbon nanotubes are known to exhibit many interesting mechanical and electrical properties due to their unique one-dimensional (1D) structures and are regarded as one of the most important materials for nanoscale device applications [1]. Recent reports of photoluminescence (PL) [2-4] from the direct band-gap semiconducting carbon nanotubes extend the possible applications to optoelectronics. Here we report onedimensional $(4,2)$ carbon nanotubes, which are semiconducting with an indirect narrow band gap $(\sim 0.2 \mathrm{eV})$ in pristine form, and metallic $(3,3)$ tubes as efficient visible PL materials, with a PL efficiency on the order of $1 \%-5 \%$.

The carbon nanotubes that emit visible PL are grown by pyrolysis of tripropylamine $\left[\left(\mathrm{CH}_{3} \mathrm{CH}_{2} \mathrm{CH}_{2}\right)_{3} \mathrm{~N}\right.$, TPA] in the channels of the host single crystalline aluminophosphate $\mathrm{AlPO}_{4}-5$ (AFI) zeolite [5,6]. These carbon nanotubes are monosized and parallel in alignment. They offer the opportunity to study the intrinsic anisotropic physical properties of 1D single walled nanotubes (SWNTs) in the form of macroscopic samples. From high-resolution transmission electron microscopy [7] and Raman scattering measurement [6,8], our SWNTs are $0.4 \mathrm{~nm}$ in diameter and can take only a limited number of possible structures: $(5,0),(3,3)$, and $(4,2)$. Two types of sample are prepared: A type I sample, containing $(5,0),(3,3)$, and $(4,2)$ tubes, is fabricated by pyrolysis of TPA@AFI crystal at $580^{\circ} \mathrm{C}$ without pumping out the hydrocarbon radicals such as $\mathrm{CH}_{3}$ and $\mathrm{C}_{2} \mathrm{H}_{5}$ that were decomposed from the TPA molecules in the zeolite channels from the reaction chamber. A type II sample, mainly containing $(5,0)$ and $(3,3)$ tubes, is fabricated in a similar way except by pumping away all the hydrocarbon radicals. These two types of sample can be distinguished through their absorption and Raman spectra.

The AFI crystals containing SWNTs used in the present work had a typical size of about $100 \mu \mathrm{m}$ in diameter and about $400 \mu \mathrm{m}$ in length and were uniform in color. In the far-field PL measurements, an $\mathrm{Ar}^{+}$laser beam at $488 \mathrm{~nm}$ was incident onto the sample with a spot size of $\sim 25 \mu \mathrm{m}$. The PL was collected at an angle of $\sim 10^{\circ}$ away from the incident beam and was dispersed by a $\frac{1}{2}$ meter monochromator, before entering a photomultiplier tube or a Ge detector. The inset in Fig. 1 shows the geometry of the far-field PL measurements, including the polarizations of the excitation laser beam and the emission light. In the near-field PL measurements, laser beams with different wavelengths were coupled into an optical fiber one at a time and directed to a tapered tip in a nearfield scanning optical microscope (NSOM). The illuminated spot size on the sample was $\sim 1 \mu \mathrm{m}$ and the PL was

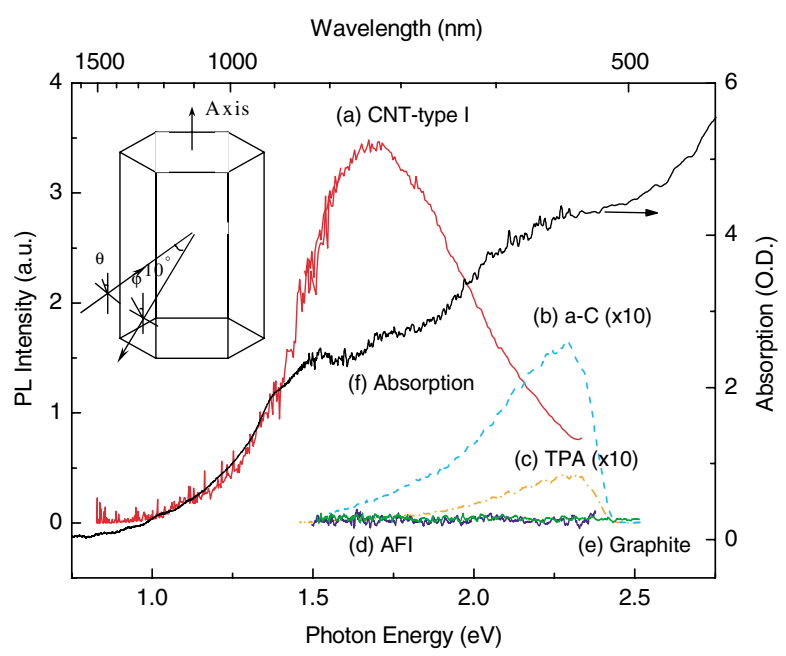

FIG. 1 (color online). (a) PL spectrum from a type I SWNT sample at room temperature when excited at $488 \mathrm{~nm}$. For comparison, the PL spectra from (b) amorphous carbon in AFI, (c) TPA in AFI, (d) pure AFI, and (e) graphite are also plotted. (f) Together is shown the absorption spectrum of the corresponding sample. The inset shows the geometry and the polarization arrangement in the far-field PL measurements. The hexagonal prism represents the AFI zeolite crystal with carbon nanotubes aligned parallel to the axis. 
collected in the far-field mode with a $1 / 4$ meter monochromator and a charge-coupled device detector. In both cases, a mercury lamp was used for calibrating the wavelength of the monochromators and a quartz-tungstenhalogen irradiation source was used for calibrating the detection sensitivities of the system at different wavelengths [9].

In Fig. 1, we show a PL spectrum (curve $a$ ) from a type I SWNT sample at room temperature from 530 to $1500 \mathrm{~nm}$ when excited by a $488 \mathrm{~nm}$ laser beam at $\theta=0^{\circ}$ and collected at $\varphi=0^{\circ}$. A broad and intense emission band is observed centered at $\sim 730 \mathrm{~nm}(1.70 \mathrm{eV})$. The overall PL efficiency has been determined as 1\%-5\%, depending on samples. We have also plotted the absorption spectrum (curve $f$ ) in Fig. 1 with incident light polarization parallel to the nanotubes in the same spectral range. While the absorption has three bands, one in the near infrared $(\sim 1.4 \mathrm{eV})$, one in the visible $(\sim 2.1 \mathrm{eV})$, and the other in UV $(\sim 3.0 \mathrm{eV}$, not shown here), the PL emission spectrum is clearly below the visible absorption band. By varying the excitation power from 0.02 to $1.6 \mathrm{~mW}$, over a dynamic range of $\sim 80$-fold, we found that the PL intensity and shape is linear to the input laser power. In order to rule out the PL contribution from other possible species [10] in the AFI crystal, the PL spectra from a TPA (precursor) contained and an amorphous carbon contained (heat treatment of TPA contained AFI sample to only $400^{\circ} \mathrm{C}$ [6]) AFI zeolite samples together with bare AFI and graphite samples are also plotted in Fig. 1. Clearly, TPA (curve $c$ ) and amorphous carbon (curve $b$ ) reveal very different spectral features both in emission wavelength and intensity from the SWNTs.

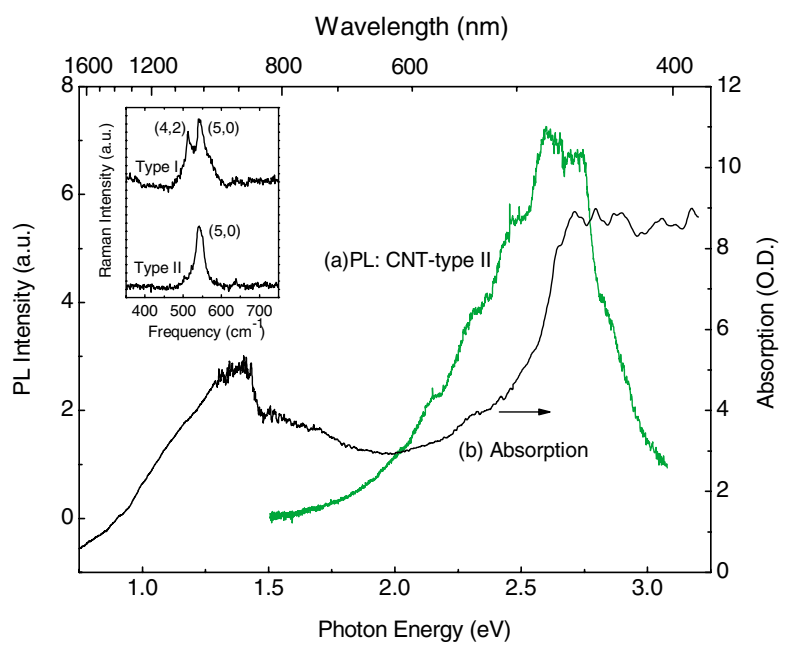

FIG. 2 (color online). (a) PL spectrum from a type II SWNT sample at room temperature when excited at $325 \mathrm{~nm}$. (b) The corresponding absorption spectrum. The PL data are taken by NSOM. The inset shows the Raman spectra of type I and type II samples at $632.8 \mathrm{~nm}$ laser excitation. $\mathrm{RBM}$ of $(4,2)$ is absent in type II sample. Without resonant enhancement, the RBM of $(3,3)$ cannot be observed in both samples.
These evidences lead to the assignment of PL at $1.7 \mathrm{eV}$ as arising from the $0.4 \mathrm{~nm}$ carbon nanotubes.

In comparison, the type II sample has absorption bands only at 1.4 and $3.0 \mathrm{eV}$, but not at $2.1 \mathrm{eV}$ (Fig. 2). When excited by a $488 \mathrm{~nm}$ laser beam, the PL at $1.7 \mathrm{eV}$ completely disappears. As shown in Fig. 2, a broad emission near $2.6 \mathrm{eV}$ is detected instead when excited by a $325 \mathrm{~nm}$ laser beam. For a type I sample, the emission at $\sim 2.6 \mathrm{eV}$ also shows up in addition to the $1.7 \mathrm{eV}$ PL peak when excited at $325 \mathrm{~nm}$ with moderate to high laser power. According to Refs. [11,12], the absorption band at $2.1 \mathrm{eV}$ is solely due to the $(4,2)$ tubes, while the 1.4 and $3.0 \mathrm{eV}$ absorptions originate from $(5,0)$ and $(3,3)$, respectively. This indicates that type I samples contain all $(4,2),(5,0)$, and $(3,3)$ tubes but type II samples contain only $(5,0)$ and $(3,3)$ tubes. The Raman spectra of the radial breathing modes (RBM) (inset in Fig. 2) further indicate that $(4,2)$ tubes are absent in type II samples [13]. Thus, the PL at $\sim 1.7 \mathrm{eV}$ shown in Fig. 1 (curve $a$ ) must have originated from the $(4,2)$ tubes. Since the absorption band at $\sim 3.0 \mathrm{eV}$ comes only from the $(3,3)$ tubes, the PL shown in Fig. 2 must be due to the $(3,3)$ tubes but not the $(5,0)$ tubes. The PL at $\sim 2.6 \mathrm{eV}$ from both type I and type II samples clearly provides strong evidence that metallic $(3,3)$ tubes emit light at a high efficiency.

In order to understand the correlation between the 1D structure of the SWNTs and the PL emission, we have measured the polarization dependence for both excitation and emission for a type I sample. Figure 3(a) shows the anisotropy in the total PL signal (no discrimination of the emission light polarization) at room temperature when the excitation polarization varies from nearly parallel to the nanotubes to perpendicular to them. The integrated PL intensity [see inset in Fig. 3(a)] is observed to decrease monotonically by about 5 times from $\theta=0^{\circ}$ (longitudinal excitation) to $\theta=90^{\circ}$ (transversal excitation) and follows a $\cos ^{2} \theta$ law. In Fig. 3(b), an analysis of the polarization of the emitted light is shown for longitudinal excitation. The emitted PL is mainly polarized along the sample axis, with the intensity of the axis-parallel component $\sim 10$ times larger than that of the axisperpendicular component. The distinctive anisotropy implies that the absorption and emission dipoles are basically along the nanotube axis, indicating that the electronic states of the nanotubes are much easier to be polarized along the nanotube than perpendicular to it. Because of the 1D nature of the carbon nanotubes, this conclusion is reasonable [14].

The large polarization dependence of the PL spectra for both excitation and emission further confirms that the PL peaked at $730 \mathrm{~nm}(1.70 \mathrm{eV})$ originates from the $0.4 \mathrm{~nm}$ carbon nanotubes. In contrast, aside from the weaker intensity and different spectral range, the PL signals from the precursor TPA molecules and the amorphous carbons in the AFI crystals have a very weak polarization dependence. Only a $\sim 20 \%$ (10\%) decrease in PL intensity is observed when the excitation (emission) polarization 

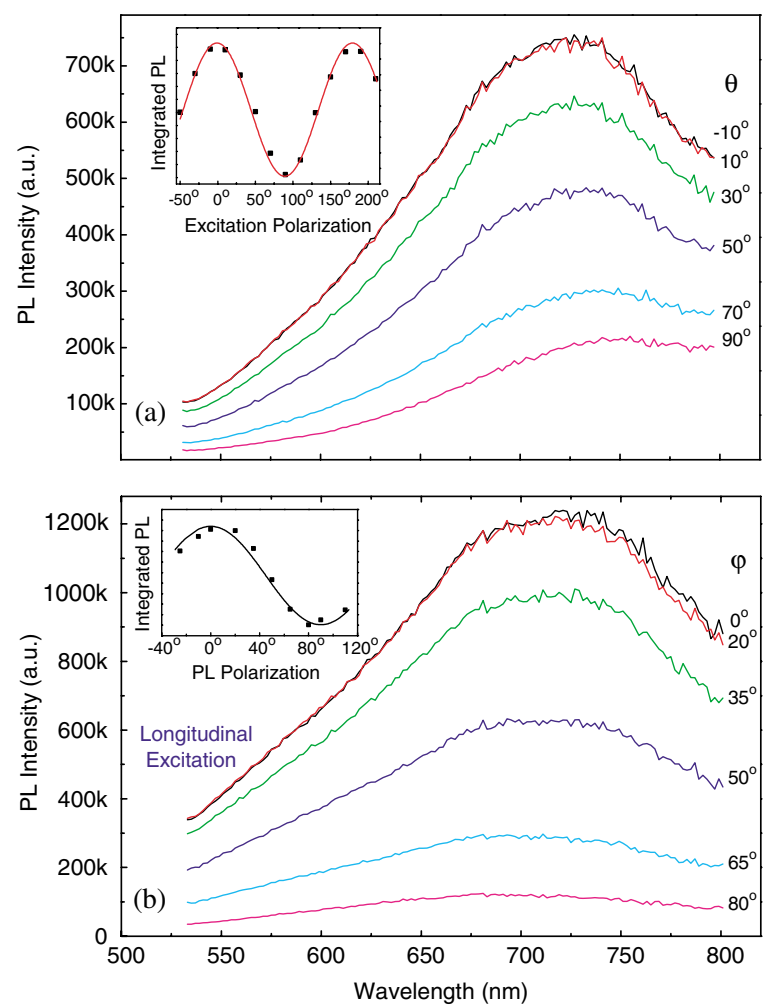

FIG. 3 (color online). (a) PL spectra for different excitation polarizations at room temperature. The solid squares in the inset show the integrated PL intensity as a function of the

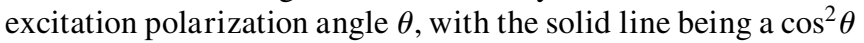
law fitting. (b) PL spectra as a function of emission light polarization angle $\varphi$ for longitudinal excitation at room temperature. The solid squares in the inset show the integrated PL intensity as a function of the emission light polarization angle $\varphi$, with the solid line being a $\cos ^{2} \varphi$ law fitting.

rotates from parallel to perpendicular to the zeolite crystal axis, consistent with their non-1D structures.

Using density functional methods [15], the band structures of the pure and nitrogen-incorporated $\left(\mathrm{C}_{55} \mathrm{~N}\right)(4,2)$ tubes together with the pure $(3,3)$ tube are calculated and shown in panels (a), (b), and (c) of Fig. 4, respectively. The band structures indicate that the pure $(3,3)$ nanotube is a metal and the pure $(4,2)$ nanotube is an indirect bandgap $(\sim 0.2 \mathrm{eV})$ semiconductor $[11,12]$. Therefore, the mechanism responsible for PL of direct band-gap semiconducting carbon nanotubes [2-4], i.e., photon emission through a recombination between the electrons in conduction band and the holes in valence band, cannot account for the observation of visible PL from $(4,2)$ and $(3,3)$ tubes.

The absorption spectra of $(4,2)$ and $(3,3)$ tubes have been explained by Refs. $[11,12,14]$. Electron excitation through a dipole allowed transition from the van Hove singularities originated from bands with $E_{10 g}$ symmetry (in red color) to bands with $E_{10 u}$ symmetry (in blue color) for $(4,2)$ tubes, and $E_{g}$ to $E_{u}$ for $(3,3)$ tubes, gives rise to an absorption band at $2.1 \mathrm{eV}$ [Fig. $4(\mathrm{a})]$ for $(4,2)$ tubes

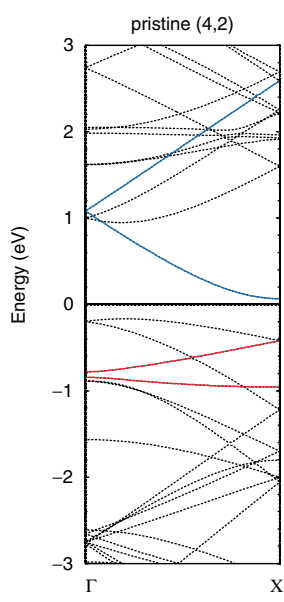

(a)

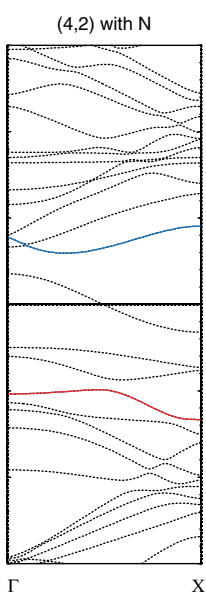

(b)

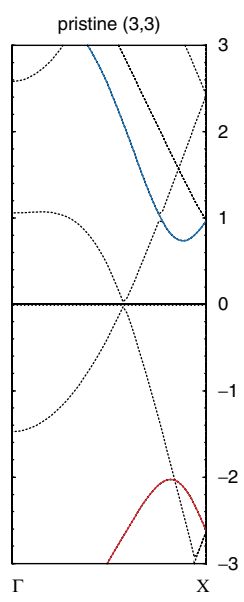

(c)
FIG. 4 (color). The band structure of pure $(4,2), \mathrm{N}$-doped $(4,2)(1.8 \%$ of carbon substituted by $N)$, and pure $(3,3)$ tubes near the Fermi level. The bands with dipole allowed optical transitions that lead to strong absorption are labeled in colors.

and at $3.0 \mathrm{eV}$ [Fig. 4(c)] for $(3,3)$ tubes, respectively. However, in contrast to the 3D case, the crossings between different bands in carbon nanotubes as a quasi-onedimensional system take place only at a few $k$ points, leading to slow interband relaxation through scatterings via such $k$ points with or without phonons. In the case of $(3,3)$ tubes, for instance, electrons relaxed to the bottom region of the blue bands and the holes relaxed to the top region of red bands would be stuck there. Dipole allowed photon emission could occur to give rise to the observed $\mathrm{PL}$ at $2.6 \mathrm{eV}$, which has a lower energy than the absorption due to Stokes shift.

In contrast, the $1.7 \mathrm{eV} \mathrm{PL}$ from $(4,2)$ tubes is more difficult to interpret. It must invoke a new mechanism that is different from the conventional band-edge emission mechanism for direct band-gap semiconductors. The $(4,2)$ has a strong absorption peak at $2.1 \mathrm{eV}$ that comes from the van Hove singularities of the bands labeled red and blue in Fig. 4(a). However, the band structure of the pristine $(4,2)$ tube, as shown in Fig. 4(a), shows that the electron (hole) can relax to lower (higher) energy bands, and the resulting PL should be $\sim 0.2 \mathrm{eV}$, rather than $1.7 \mathrm{eV}$, unless there is some new mechanism that suppresses interband and intraband relaxations. A plausible explanation is that the tubes contain substitutional defects such as $\mathrm{N}$. Two reasons support the $\mathrm{N}$-doping hypothesis: one is that the precursor TPA molecules, $\left(\mathrm{CH}_{3} \mathrm{CH}_{2} \mathrm{CH}_{2}\right)_{3} \mathrm{~N}$, have a fairly large percentage of $\mathrm{N}$. The other is that the $\mathrm{x}$-ray photoemission spectroscopy signal from these carbon nanotube samples with or without the zeolite matrix indicates the existence of $\sim 2 \% \mathrm{~N}$ in the aromatic form. With $1 \mathrm{~N}$ substituting a $\mathrm{C}$ atom per unit cell of 56 atoms in $(4,2)$ tubes, the resultant $\mathrm{C}_{55} \mathrm{~N}$ tube $(1.8 \% \mathrm{~N})$ has energy band structures shown in Fig. 4(b). The effect of the $\mathrm{N}$ substitution is twofold: (i) The additional electron from the $\mathrm{N}$ atom raises the Fermi level, making the tube 
metallic. (ii) The defect breaks the symmetry of the tube, leading to repulsion between crossing bands and thereby creating gaps. Such avoided band crossings in one direction would hardly be of any consequence in 3D metals, but in 1D systems, the minigaps would block the only available relaxation channels. This is indeed found to be the case in Fig. 4(b), where the blue and red bands now give rise to van Hove singularities that electrons and holes cannot further relax towards the Fermi level. We note that while Fig. 4(b) pertains to an idealized ordered structure of a particular $\mathrm{N}$ substitution concentrations, it is reasonable to expect that randomly positioned substitutional impurities would have similar effects of minigap opening due to symmetry breaking, which may serve to block the very limited channels of intraband relaxations in these quasi-1D systems. The energy difference between the two dipole allowed transition bands in Fig. 4(b) matches well with the $1.7 \mathrm{eV}$ PL peak and thus provides a plausible explanation to $(4,2)$ tubes. The broad PL width may arise from the nonuniform $\mathrm{N}$ doping and the large Stokes shift associated with energy transfer from the pure carbon part to the $\mathrm{N}$-doped part of the tubes.

Our above interpretation makes use of the band structures for $(3,3)$ and $\mathrm{N}$-doped $(4,2)$ tubes and assumes a lower nonradiative recombination rate for electrons and holes to relax to the Fermi level due to limited relaxation pathways offered by the $1 \mathrm{D}$ band structure in contrast to 3D case. While this proposed mechanism can explain well the peak positions and the polarization dependence for the emission from both $(3,3)$ and $(4,2)$ tubes, the $\mathrm{N}$-doping assumption based on circumstantial evidence rather than a definitive microscopic characterization remains as its weak point.

Other defect states such as tube end states may provide a second possible mechanism for PL. As indicated by some new calculations by Louie [16], other defects may exist and provide density of states in the band gap. In particular, it is worth pointing out that the carbon nanotubes produced in the zeolite matrix are not ideal; e.g., the tubes have finite lengths and a length distribution [17]. At this stage, we have little knowledge on the nature of these end states and other various kinds of defect state in terms of their energy levels, transition dipole moments, etc. Assuming that these defect states exist, either due to different configurations of the defects or due to the interaction between the end atoms and atoms within the tubes in various lengths, a range of energy levels may result, which can thus account for the PL peak position as well as the broad spectral width. However, it is not easy for this mechanism to explain the observed strong polarization dependence.

In summary, we have observed efficient PL from $(4,2)$ and $(3,3)$ tubes, which has a strong polarization effect in both excitation and emission. We have proposed two plau- sible mechanisms to explain our observation: one involves a suppression of nonradiative decay due to the one-dimensionality of the carbon nanotubes' band structures, and the other involves defect states such as end states of the carbon nanotubes.

The authors thank Professors Ping Sheng, Micheal Loy, Steven Louie, Peter Yu, and Riichiro Saito for fruitful discussions. We acknowledge the financial support of the Hong Kong University of Science and Technology through the William Mong Solid State Cluster Laboratory, and the High Impact Area Fund and the Research Grant Council of Hong Kong through Projects No. HKUST6134/97P, No. HKUST6057/02P, and No. HKUST6152/01P.

*To whom correspondence should be addressed. Electronic address: phxudong@ust.hk

[1] Carbon Nanotubes: Synthesis, Structures and Applications, edited by M.S. Dresselhaus, G. Dresselhaus, and $\mathrm{Ph}$. Avouris (Springer, Berlin, 2001).

[2] M. J. O'Connell et al., Science 297, 593 (2002).

[3] S. M. Balhilo et al., Science 298, 2361 (2002).

[4] J. Lefebvre, Y. Homma, and P. Finnie, Phys. Rev. Lett. 90, 217401 (2003).

[5] Z. K. Tang et al., Appl. Phys. Lett. 73, 2287 (1998).

[6] H. D. Sun et al., Appl. Phys. A 69, 381 (1999).

[7] N. Wang et al., Nature (London) 408, 50 (2000).

[8] A. Jorio et al., Chem. Phys. Lett. 351, 27 (2002).

[9] K. D. Mielenz, Measurement of Photoluminescence (Academic, New York, 1982).

[10] From the mass spectroscopy of the species outgassed from the nanotube-zeolite sample and the Raman spectroscopy, we conclude that there are no hydrocarbon groups and thus no other organic species in our samples.

[11] Z. M. Li et al., Phys. Rev. Lett. 87, 127401 (2001).

[12] H. J. Liu and C. T. Chan, Phys. Rev. B 66, 115416 (2002).

[13] M. Hulman et al., J. Chem. Phys. 119, 3384 (2003).

[14] L. X. Benedict, S. G. Louie, and M. L. Cohen, Phys. Rev. B 52, 8541 (1995); M. Machón et al., Phys. Rev. B 66, 155410 (2002); W. Z. Liang et al., Appl. Phys. Lett. 80, 3415 (2002).

[15] To model the nitrogen impurities in these tubes, we replace one carbon atom by nitrogen in the supercells containing five primitive cells for $(3,3)$ and one primitive cell for $(4,2)$ and the nominal formulas are, respectively, $\mathrm{C}_{59} \mathrm{~N}$ for $(3,3)$ and $\mathrm{C}_{55} \mathrm{~N}$ for $(4,2)$. The tubes in the supercells are aligned in a hexagonal array with the closest distance between adjacent tubes being $10 \AA$. We use a plane-wave pseudopotential approach and the Perdew-Wang-91 generalized gradient corrections. All atomic degrees of freedom are fully relaxed. We found that it is about $0.5 \mathrm{eV}$ more favorable for $\mathrm{N}$ to substitute a carbon site in $(4,2)$ than in $(3,3)$.

[16] S. G. Louie (private communication).

[17] Z. K. Tang et al., Science 292, 2462 (2001). 